\title{
INFRARED TECHNIQUES FOR COMET OBSERVATIONS
}

\author{
MARTHA S. HANNER \\ Jet Propulsion Laboratory \\ California Institute of Technology \\ 4800 Oak Grove Drive \\ Pasadena, CA 91109
}

\author{
ALAN T. TOKUNAGA \\ Institute for Astronomy \\ University of Hawaii \\ 2680 Woodlawn Drive \\ Honolulu, HI 96822
}

\begin{abstract}
The infrared spectral region $(1-1000 \mu \mathrm{m})$ is important for studies of both molecules and solid grains in comets. Infrared astronomy is in the midst of a technological revolution, with the development of sensitive 2-dimensional arrays leading to infrared cameras and spectrometers with vastly improved sensitivity and resolution. The Halley campaign gave us tantalizing first glimpses of the comet science possible with this new technology, evidenced, for example, by the many new spectral features detected in the infrared. The techniques of photometry, imaging, and spectroscopy are reviewed in this chapter and their status at the time of the Halley observations is described.
\end{abstract}

\section{Introduction}

The infrared spectral region $(\lambda 1-1000 \mu \mathrm{m}$ ) is vital to understanding the composition of comets and the chenistry of the inner coma, since many molecules and mineral phases have fundamental bands and rotational lines in the infrared. There has been much progress in infrared techniques since the first infrared detection of Comet Ikeya-Seki (1965f) at $\lambda 1.65$ $10 \mu \mathrm{m}$ by Becklin and Westphal (1966). Halley was the first comet to be studied with a wide range of modern infrared instrumentation, both ground-based and airborne. Exciting new discoveries, such as the 3.36- $\mu \mathrm{m}$ emission from unknown organic species, resulted from infrared observations of Comet Halley, and more than 20 infrared spectral features were detected, most for the first time (Campins and Tokunaga 1988). Small dust grains radiate thermal emission primarily between 3-20 $\mu \mathrm{m}$, while emission from larger grains can be detected at longer wavelengths. The abundance, size, and spatial distribution of dust grains in the coma can best be assessed via their thermal emission. Silicates, a major component of the grains, have features at 8-12 $\mu \mathrm{m}$ and $16-22 \mu \mathrm{m}$. Finally, in a few low-activity comets, thermal emission from the nucleus can be directly detected.

Today, infrared astronomy is changing at a rapid rate, with the advent of large, sensitive 2-dimensional arrays. This new technology makes possible IR cameras and spectrometers with a sensitivity and resolution only dreamed of a few years ago. Halley's apparition took place on the threshold of this new era. Because of the rapid changes in infrared techniques, we have decided to summarize the status of the field at the time of IIalley, as an aid to future rescarchers who will use the Halley archive produced by the International Halley Watch. 
The emphasis in this paper is on techniques for infrared comet observations. While we highlight the science achievable with each technique, it is not our purpose to review the body of science resulting from these observations, nor to chronicle the extensive Halley observations. We refer readers to the report of the 1987 workshop on infrared observations of Halley (Hanner 1988), to the many papers in Astronomy and Astrophysics 187, 1987, and to other papers in this volume.

\section{Photometry}

\subsection{SCIENCE GOALS}

Infrared photometry of comets measures primarily the radiation from the small dust particles in the coma. Since the black body temperature near $1 \mathrm{AU}$ is $\sim 300 \mathrm{~K}$, comet dust has its peak thermal emission near $10 \mu \mathrm{m}$, and a major portion of the thermal energy distribution is accessible to ground-based observers. In the near infrared $(\lambda<2.5 \mu \mathrm{m})$, scattered radiation from the dust is observed. In a few cases where the comet activity is low, the nucleus can be directly detected in the infrared.

The major science goals for comet IR photometry can be summarized as follows:

1. Define the spectral energy distribution of the thermal emission from the dust grains between 3.5 and $20 \mu \mathrm{m}$.

2. Determine the arnount of dust in the coma and the dust production rate.

3. Detect silicate emission near 10 and $20 \mu \mathrm{m}$ and measure its strength relative to the continuum.

4. Detect the nuclei of low-activity comets and determine their size, shape, albedo, and surface properties.

5. Measure the near-infrared $(1-2.5 \mu \mathrm{m})$ colors of the scattered radiation from the dust for comparison to other solar system bodies such as asteroids and rings.

6. Monitor long- and short-term temporal variations in all of the above quantities.

Ney (1982) reviewed IR photometry of the bright comets in the 1970s. Since then, a number of fainter, short-period comets have been observed (cf. Hanner 1984). The infrared observations of Comet Halley have been summarized by Campins and Tokunaga (1988). Their Table 1.1 lists the Halley infrared photometric monitoring programs. The main infrared characteristics of the dust in all of the comets are a thermal energy distribution broader than that of a single black body; a color temperature higher than that of a black body in equilibrium; JHK colors slightly red relative to the Sun; and a $10-\mu \mathrm{m}$ silicate emission feature in most comets within $1 \mathrm{AU}$ of the sun.

Because comet nuclei have turned out to be dark and warm, thermal infrared photometry is a powerful tool for remote study of the size, shape, and surface thermal properties of comet nuclei. A recent review is given by A'Hearn (1988). 


\subsection{PHOTOMETRIC SYSTEMS}

Photometry is the calibrated measurement of the irradiance of an object in a defined spectral bandpass. A photometric system consists of one or more spectral bandpasses defined by a) filter + detector responsivity, b) an absolute flux calibration, and c) a net of standard stars whose magnitudes are accurately known relative to the flux standard.

Unfortunately, a single, common infrared photometric system does not exist today, to the frustration of those who attempt to compare and interpret comet observations. Nevertheless, considerable progress has been made during the $1980 \mathrm{~s}$, and we believe that the goal of a single accurate absolute calibration and standard star net from $1-20 \mu \mathrm{m}$ is within reach. In this section we discuss the elements of the photometric systems and their status at the time of Halley observations (1985-1987).

2.2.1 Detectors. The detectors used for infrared photometry are described by Low and Rieke (1974), Gillett et al. (1977), and Gehrz et al. (1987), among others. The galliumdoped germanium bolometer (Low 1961) is almost universally used at wavelengths between 5 and $25 \mu \mathrm{m}$, because of its uniform sensitivity; it is background-limited at these wavelengths. The temperature of the germanium element, which changes in response to the incident infrared flux, is measured via the change in the element's electrical resistance.

Photoconductors, made from doped Ge or Si crystals, are more sensitive than bolometers and are therefore preferred for low-background applications, such as (CVF) spectrometers, grating spectrometers, or airborne and space-based instruments. The doping material, such as arsenic or bismuth, causes an energy level in the crystal to be created such that infrared photons can inject electrons into the conduction band. By placing a voltage across the photoconductor, a current can be generated that is proportional to the infrared illumination.

At $1-5 \mu \mathrm{m}$, the InSb photovoltaic detector is most commonly used. Operating like a solar cell, the photovoltaic detector generates a current proportional to the infrared illumination. Another type of photovoltaic detector is $\mathrm{HgCdTe}$, which has a long-wavelength cutoff that is dependent on the ratio of $\mathrm{Hg}$ to $\mathrm{Cd}$. Two-dimensional arrays of $1-$ to $2.5-\mu \mathrm{m}$ $\mathrm{HgCdTe}$ detectors are coming into use at the present time (see Section 3). Arrays of 8 - to $14-\mu \mathrm{m} \mathrm{HgCdTe}$ detectors are manufactured, but are not commonly used in astronomy.

2.2.2 Spectral Bandpasses. Ground-based infrared photometry is limited to spectral "windows," where the atmospheric transmission is high (Fig. 1). Johnson (1965b, 1966) established a wide-band photometric system for the infrared, tailored to the atmospheric windows. The systems in use today derive from the Johnson system, although the filter characteristics differ somewhat at various observatories, even for the so-called standard bandpasses. The standard bandpasses and their letter designations are summarized in Table 2-1. Intermediate bandpass filters $(\lambda / \Delta \lambda \sim 10)$, tailored to specific astronomical studies such as the $10-$ and $20-\mu \mathrm{m}$ silicate features or the $3-\mu \mathrm{m}$ ice band, are also widely used. A filter at $18 \mu \mathrm{m}$, to take advantage of the cleanest part of the $10-\mu \mathrm{m}$ atmospheric window, has been used for comet observations (Ney 1974, 1982).

A spectral bandpass is described by an effective wavelength and a band width (full width at half-maximum). The effective wavelength is strictly defined only by the instrument response function $\phi(\lambda)$ (King 1952, Johnson 1965a). 


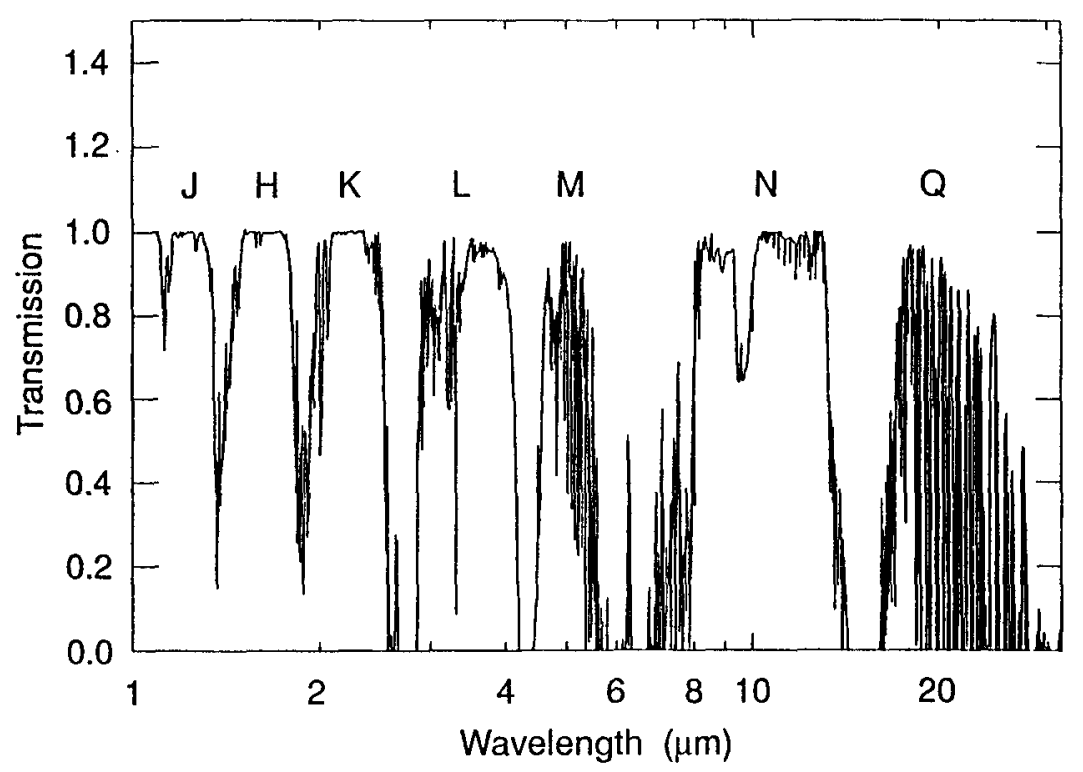

Fig. I Atmospheric Transmission (courtesy of Charles Lindsey)

Table 2-1. Standard Infrared Photometric Bandpasses

\begin{tabular}{lcccccccc}
\hline & \multicolumn{4}{c}{ Arizona System } & \multicolumn{5}{c}{ IRTF System } \\
Filter & Johnson & $(1965)$ & Campins $e$ al. $(1985)$ & \multicolumn{5}{c}{ Tokunaga (1986) } \\
& $\lambda_{o}$ & $\Delta \lambda$ & $\lambda_{o}$ & $\Delta \lambda$ & $\lambda_{o}$ & $\Delta \lambda$ & $\lambda_{s}{ }^{c}$ & $\lambda_{\text {ref }}$ \\
& $\mu \mathrm{m}$ & $\mu \mathrm{m}$ & $\mu \mathrm{m}$ & $\mu \mathrm{m}$ & $\mu \mathrm{m}$ & $\mu \mathrm{m}$ & $\mu \mathrm{m}$ & $\mu \mathrm{m}$ \\
\hline $\mathrm{J}$ & 1.25 & 0.37 & 1.26 & 0.20 & 1.20 & 0.3 & & 1.25 \\
$\mathrm{H}$ & - & - & 1.60 & 0.36 & 1.6 & 0.3 & & 1.65 \\
$\mathrm{~K}$ & 2.2 & 0.6 & 2.22 & 0.52 & 2.2 & 0.4 & & 2.2 \\
$\mathrm{~L}$ & $3.5^{\mathrm{a}}$ & 1.0 & 3.54 & 0.97 & 3.55 & 1.05 & 3.46 & 3.45 \\
$\mathrm{~L}^{\prime}$ & - & - & - & - & 3.78 & 0.57 & 3.75 & 3.80 \\
$\mathrm{M}$ & 5.0 & 1.2 & 4.80 & 0.60 & 4.7 & 0.57 & 4.70 & $4.7^{\mathrm{d}}$ \\
$\mathrm{N}$ & 10.2 & 5.6 & $10.6^{\mathrm{b}}$ & 5. & 10.50 & 5. & 9.8 & 10.1 \\
$\mathrm{Q}$ & - & - & $21.0^{\mathrm{b}}$ & 11.0 & 20.6 & 9. & 19.6 & 20.0 \\
\hline
\end{tabular}

$\lambda_{o}=\int \lambda \phi(\lambda) d \lambda / \int \phi(\lambda) d \lambda$, where $\phi(\lambda)=$ filter transmission at $77 \mathrm{~K}$.

$\Delta \lambda=$ full width at half-maximum

$\lambda_{s}=$ weighted by stellar flux at $6000 \mathrm{~K}$; see Eq. 2

$\lambda_{\text {ref }}=$ reference wavelength for monochromatic flux; see text

a for InSb detector

${ }^{\mathrm{b}}$ Rieke et al. (1985); Low and Rieke (1974)

c includes sky transmission

${ }^{d}$ revised for cooled filter 


$$
\lambda_{e f f}=\int \lambda \phi(\lambda) d \lambda / \int \phi(\lambda) d \lambda
$$

The filter transmission needs to be known at the operating temperature; a shift of $1.5 \%$ towards shorter $\lambda$ at $77 \mathrm{~K}$ compared with that at room temperature is typical.

It is often useful to include the atmospheric emission, $\tau(\lambda)$, and the energy distribution of the source, $S(\lambda)$, to define a wavelength

$$
\lambda_{s}=\int \lambda S(\lambda) \tau(\lambda) \phi(\lambda) d \lambda / \int S(\lambda) \tau(\lambda) \phi(\lambda) d \lambda
$$

Low and Rieke (1974) show that the $\lambda_{s}$ at $Q$ depends on the atmospheric water vapor content, shifting by more than $1 \mu \mathrm{m}$ towards shorter wavelength for high extinction.

In the infrared, on the Rayleigh-Jeans tail of the stellar spectral energy distribution, the $\lambda_{s}$ is essentially the same for stars of all spectral types, but can vary by several tenths of a micrometer for comets, where the dust temperatures range from less than $200 \mathrm{~K}$ to greater than $600 \mathrm{~K}$.

Data are frequently quoted as the flux equivalent to the monochromatic flux at some reference wavelength, $\lambda_{r}$. The monochromatic flux, $\mathrm{S}\left(\lambda_{r}\right)=\mathrm{f} \bar{S}$, where

$$
\bar{S}=\int S(\lambda) \tau(\lambda) \phi(\lambda) d \lambda / \int \tau(\lambda) \phi(\lambda) d \lambda
$$

is the quantity actually measured at the telescope and $\mathrm{f}$ is a correction factor that depends on the spectral shape of the source and the reference wavelength. In practice, the correction factor has to include the difference in spectral shape between the comet and the standard stars. Hanner et al. (1984) and Tokunaga (1986) took the approach of choosing $\lambda_{r}=10.1$ $\mu \mathrm{m}$ at $\mathrm{N}$ and $20 \mu \mathrm{m}$ at $\mathrm{Q}$ to minimize the correction factors [IRTF system], while Low and Rieke selected $\lambda_{r}$ of $10.6 \mu \mathrm{m}$ and $21 \mu \mathrm{m}$, closer to the effective wavelength [Arizona system].

When comparing comet observations taken with wide bandpasses, one should note the reference wavelengths and correction factors. Corrections are generally not necessary for filter bandpasses having $\lambda / \Delta \lambda>10$. Filter transmission curves will be included in the Halley archive.

2.2.3 Absolute Calibration. Direct absolute flux calibration requires transfer of a laboratory absolute flux standard via a chain of direct measurements to one or more standard stars. While direct calibrations of Vega at visual wavelengths $(\lambda \leq 1.04 \mu \mathrm{m})$ have achieved an accuracy of $1 \%$ to $1.5 \%$ (Hayes 1985), the process is inherently more difficult in the infrared, and indirect methods have often been adopted. A direct calibration of Vega versus a standard furnace mounted to the telescope has been carried out between 1-5 $\mu \mathrm{m}$ by Blackwell et al. (1983), Mountain et al. (1985) and Booth et al. (1989). Their estimated accuracy is $3 \%$ at $1-4 \mu \mathrm{m}$ and $8 \%$ near $5 \mu \mathrm{m}$.

Campins et al. (1985) applied the solar analog method to calibrate the JHKLM bandpasses on the Arizona photometric system. They observed several solar analog stars through these filters to establish the solar color, then used the solar spectrum from Neckel and Labs (1981) and the solar visual magnitude $V=-26.76$ to compute the absolute flux density of the Sun in each of the filter bandpasses and the corresponding flux for Vega. Their results agree with Blackwell et al. and Mountain et al. to about $5 \%$. Thus, the average of the two 
Table 2-2. Absolute Flux Density of Vega ( $\alpha$ Lyrae)

\begin{tabular}{ccccc}
\hline Filter Band & $\begin{array}{c}\lambda_{\mathrm{r}} \\
\mu \mathrm{m}\end{array}$ & $\begin{array}{c}\mathbf{f}(\lambda) \\
\mathrm{Wm}^{-2} \mu \mathrm{m}^{-1}\end{array}$ & $\begin{array}{c}\mathrm{f}(\nu) \\
\mathrm{Wm}^{-2} \mathrm{~Hz}^{-1}\end{array}$ & Ref. \\
\hline $\mathrm{J}$ & 1.26 & $3.00 \times 10^{-9}$ & $1.570 \times 10^{-23}$ & 1 \\
$\mathrm{H}$ & 1.60 & $1.24 \times 10^{-10}$ & $1.055 \times 10^{-23}$ & 1 \\
$\mathrm{~K}$ & 2.20 & $4.07 \times 10^{-10}$ & $6.570 \times 10^{-24}$ & 1 \\
$\mathrm{~L}$ & 3.54 & $6.77 \times 10^{-11}$ & $2.830 \times 10^{-24}$ & 1 \\
$\mathrm{~L}^{\prime}$ & 3.80 & $5.24 \times 10^{-11}$ & $2.520 \times 10^{-24}$ & 2 \\
$\mathrm{M}$ & 4.80 & $2.17 \times 10^{-11}$ & $1.670 \times 10^{-24}$ & 1 \\
$\mathrm{~N}$ & 10.10 & $1.17 \times 10^{-12}$ & $3.980 \times 10^{-25}$ & 3 \\
$\mathrm{Q}$ & 20.00 & $7.80 \times 10^{-14}$ & $1.040 \times 10^{-25}$ & 3 \\
\hline 1 Campins et al. $(1985)$ & & \\
2 Tokunaga (1986) & & \\
3 Rieke et al. (1985), interpolated to $\lambda_{\mathrm{r}}$ &
\end{tabular}

methods, adopted by Campins et al. as the basis for the Arizona system, should be accurate to better than $5 \%$. Both the direct and solar analog results indicate that Vega is several percent brighter than predicted by atmospheric models of Kurucz (1979) and Dreiling and Bell (1980). Tokunaga (1986) [IRTF system] averaged the direct measurements of Blackwell et al. and Mountain et al. and the solar analog determinations of Campins et al. and Wamsteker (1981), weighted according to their stated errors. The agreement with Campins et al. is within $3 \%$.

The absolute calibration at 10 and $20 \mu \mathrm{m}$ has been reviewed by Rieke et al. (1985). In 1978 , simultaneous observations of Mars were made in A rizona through the standard $\mathrm{N}$ and $\mathrm{Q}$ filters and from the Viking IRTM. This direct calibration via Mars at $10 \mu \mathrm{m}$ is in excellent agreement $(2 \%)$ with indirect calibration via the Sun and solar-type stars; however, both are about $10 \%$ higher than predicted by atmospheric models for Vega.

At $20 \mu \mathrm{m}$, the direct calibration of Mars agrees to $6 \%$ with atmospheric models of Vega normalized to the direct calibration at $10 \mu \mathrm{m}$. Since the $20-\mu \mathrm{m}$ Mars calibration has an estimated uncertainty of $8 \%$ due to the difficult correction for atmospheric absorption, Rieke et al. recommend use of the Vega model atmosphere to extrapolate from the direct $10-\mu \mathrm{m}$ calibration, although the accuracy of these models is uncertain.

Our recommended flux density for $\alpha$ Lyrae is given in Table 2-2.

Calibration at longer infrared wavelengths is more difficult, because stars such as Vega are too faint to be practical standards. For airborne observations at $20-160 \mu \mathrm{m}$, Mars is frequently used as a flux standard. This was the case for Halley measurements (Glaccum et al. 1987, Campins et al. 1987). The $\lambda$-dependent brightness temperature of Mars has to be computed for the date of observation; it can vary by $20 \%$ as a function of Sun-Mars-Earth geometry. Observers have generally used the model of Wright (1976). The model does not include the effects of dust storms! The Moon and the brightest stars such as $\alpha$ Ori are also sometimes used for calibration (Herter et al. 1987).

Absolute calibration of the $12-\mu \mathrm{m}$ IRAS bandpass is based on Rieke et al.'s flux density of $\alpha$ Tau. This calibration was extrapolated to the $25-$ and $60-\mu \mathrm{m}$ bandpasses via stellar 
models (Beichman et al. 1985). However, the 25- $\mu \mathrm{m}$ magnitude of $\alpha$ Tau differs by $7 \%$ from that of Rieke et al. (1985) [who also used stellar models] and Tokunaga (1984), after adjustment for the different zero-points. This discrepancy is not yet explained. The $100-\mu \mathrm{m}$ calibration is based on asteroid thermal models, normalized at $60 \mu \mathrm{m}$. We can look to future infrared satellites, such as COBE, ISO and SIRTF, to help improve the long-wavelength calibration and standard star net.

\subsection{STANDARD STARS}

2.3.1 Standard Stars at JHK. Comets are generally observed through the wideband J, H, and $\mathrm{K}$ filters. In this spectral region, one observes primarily the solar radiation scattered by the dust coma. Only at $r \leq 1 \mathrm{AU}$ does thermal emission from the dust begin to contaminate the $\mathrm{K}$ bandpass.

Standard star systems for $\mathrm{J}, \mathrm{H}$, and $\mathrm{K}$ have been developed at several observatories (Table 2-3). Transformations among these systems, however, have not been fully worked out. Consequently, most JHK comet observations have been published on the observer's instrumental system, meaning that it is difficult or impossible to intercompare comet colors measured with different instruments. In particular, the J filters differ in $\lambda_{\text {eff }}$ by $0.05 \mu \mathrm{m}$ or more, causing rather large color terms in the transformations (cf. Allen and Cragg 1983, Elias et al. 1983, Carter 1990).

Table 2-3. Near-Infrared Standard Star Nets

\begin{tabular}{lll}
\hline Reference & Observatory & Bandpasses \\
\hline Allen \& Cragg (1983) & AAO & JHKL' \\
Campins et al. $(1985)$ & Arizona & JHKL \\
Johnson $(1965,1966)$ & Arizona & JKLM \\
Elias et al. $(1982)$ & CTIO, CIT & JHKL \\
Engels et al. $(1981)$ & ESO & JHKLM \\
Carter (1990) & S. Africa & JHKL \\
Jones \& Hyland (1982) & Mt. Stromlo & JHKL \\
Koornneef (1983) & ESO & JHKLM \\
Sinton \& Tittemore (1984) & MKO & L'M \\
Wamsteker (1981) & ESO & JHKLM \\
\hline
\end{tabular}

In this regard, the Southern Hemisphere observatories have done better than their Northern Hemisphere counterparts. Elias et al. (1983) have published transformations between the Caltech/Cerro Tololo/Las Campanas system and the Mt. Stromlo/Anglo Australian Observatory (AAO) system, while Koornneef (1983) has compared and merged the standards from South Africa (Glass 1974), European Southern Observatory (Engels et al. 1981, Wamsteker 1981) and Arizona. S. Hemisphere (Johnson et al. 1966). Recently, Carter (1990) has published a list of 245 standards on the SAAO (South Africa) system, along with transformations to CTIO (Elias et al. 1982, 1983), ESO (Engels et al. 1981), AAO (Allen and Cragg 1983) and Mt. Stromlo (Jones and Hyland 1982). The zero-points for this system 
were defined independently, based on 25 main-sequence $\mathrm{B}$ and $\mathrm{A}$ stars, by requiring plots of $\mathrm{V}-\mathrm{K}$ vs $\mathrm{B}-\mathrm{V}, \mathrm{J}-\mathrm{K}$ vs $\mathrm{B}-\mathrm{V}$, and $\mathrm{J}-\mathrm{H}$ vs $\mathrm{B}-\mathrm{V}$ to pass through the origin. This system supersedes that of Glass (1974) and has been in use at the SAAO since 1984. Unfortunately, there is not a direct comparison between the new SAAO system and the ESO system of Koornneef (1983).

Standardization is hampered by the fact that the original Johnson system did not include the $\mathrm{H}$ filter. The zero-point for the $\mathrm{H}$ magnitude is usually defined by requiring that the slope of J-H vs H-K for the standard stars pass through the origin (Glass 1974, Wamsteker 1981, Koornneef 1983), while Carter used J-H vs B-V. Campins et al. (1985) explicitly set $\mathrm{J}-\mathrm{H}=\mathrm{H}-\mathrm{K}=\mathbf{0}$ for Vega (magnitude +0.02 at all $\lambda$ ) to define the Arizona system.

No systematic comparison between the Arizona and Mauna Kea systems has been carried out, nor between the present Northern and Southern Hemisphere systems. The J,H,K filters used in the facility InSb systems at the NASA Infrared Telescope Facility, United Kingdom Infrared Telescope, and University of Hawaii telescopes at Mauna Kea were purchased from Optical Coating Laboratory, Inc. (OCLI) in the same lot; the filters at AAO were also from the same lot (Allen and Cragg 1983). Comet observations at the IRTF have usually been referenced to standards from Elias et al. (1982), but this list contains both A and M stars.

Table 2-4. Solar Colors in Several Photometric Systems

\begin{tabular}{lllll}
\hline System & $\mathrm{J}-\mathrm{H}$ & $\mathrm{H}-\mathrm{K}$ & $\mathrm{K}-\mathrm{L}$ & References/Comments \\
\hline Arizona & 0.310 & 0.060 & 0.034 & Campins et al. (1985) 16 GV stars \\
IRTF & 0.317 & 0.072 & & A'Hearn et al. (1984) 16CygB, HD105590 \\
AAO & 0.315 & 0.065 & & Allen \& Cragg (1983) 24 G1-G5 stars \\
ESO & 0.330 & 0.044 & 0.074 & Wamsteker (1981) HD28099, HD44594 \\
ESO & 0.30 & 0.070 & 0.030 & Koornneef (1983b), transformed to Johnson system \\
\hline
\end{tabular}

Since one is interested in the color of the comet dust relative to the Sun, it is desirable to use solar-type standards. Solar analogs have been observed on several systems; the solar colors on these systems are compared in Table 2-4. Campins et al. (1985) published magnitudes of 16 solar analogs measured on the Arizona system. Observations of solar analogs are especially useful for relating comet photometry with non-standard filters.

2.3.2 Standards at 3 to $4 \mu \mathrm{m}$. Johnson (1965b) defined the L bandpass $\left(\lambda_{e f f}=3.5 \mu \mathrm{m}\right.$, FWHM $=1 \mu \mathrm{m}$ with InSb detector) to cover the 3- $\mu \mathrm{m}$ atmospheric window. Campins et al. (1985) adjusted the zero point of the Arizona system by $+0.04 \mathrm{mag}$ and presented $\mathrm{L}$ magnitudes for 14 primary standards and 12 solar analogs. Elias et al. (1982) published L magnitudes for many of their JHK standards on the CIT/CTIO system. Koornneef (1983) transformed the Southern Hemisphere observations of Glass (1974), Johnson (1966), Engels et al. (1981) and Wamsteker (1981) onto a common system that is essentially the Arizona system with the same $+0.04 \mathrm{mag}$ shift in zero point as that adopted by Campins et al.. Carter (1990) includes L magnitudes for 173 stars and presents transfers to the ESO, AAO, and CTIO systems. Carter defines the $\mathrm{L}$ zero point by requiring the slope of $\mathrm{J}-\mathrm{L}$ vs $\mathrm{B}-\mathrm{V}$ for 25 early-type standards to pass through the origin. 
For comets at $0.9<\mathrm{r}<2 \mathrm{AU}$, the $\mathrm{L}$ bandpass covers the crossover between scattered and thermal radiation from the dust. It is difficult to separate these components, since the thermal emission depends on the unknown abundance of hot submicron grains, and the scattered light albedo is wavelength-dependent. When thermal emission dominates, there is a large correction for the difference in spectral shape between comet and standard stars (Hanner et al. 1984, Tokunaga 1986). Moreover, the effective wavelength of the L filter at different observatories varies from 3.4 to $3.7 \mu \mathrm{m}$ and the FWHM from 1.2 to $0.6 \mu \mathrm{m}$. While these differences may have only a small effect on stellar colors, a shift in $\lambda_{\text {eff }}$ can have a drastic effect on the comet colors, because the thermal radiation has such a steep slope in this $\lambda$ interval. The $L$ bandpass also includes the $3.36-\mu \mathrm{m}$ emission feature discovered in Halley, but is too broad to be much help in verifying the presence of the feature.

More practical for comet photometry is the L' filter, at $\lambda_{e f f}=3.8 \mu \mathrm{m}$, FWHM $0.6 \mu \mathrm{m}$ (Allen and Cragg 1983, Tokunaga 1986). Not only is it in a cleaner part of the atmospheric window, but the thermal emission is stronger compared with the scattered light and the correction for the difference between standards and comets is smaller and more certain. Two sets of standards have been published for L': on the AAO system by Allen and Cragg and on the MKO system by Sinton and Tittemore (1984). The filters in use at AAO and Mauna Kea are apparently from the same lot at OCLI.

2.3.3 Standard Star Systems at $M(5 \mu \mathrm{m})$. The $5-\mu \mathrm{m}$ region can be observed with either bolometers or InSb detectors. Sinton and Tittemore (1984) published M magnitudes for standards on the UH/MKO InSb system. From 6 stars in common, they computed the transformation between their system and the ESO system (Engels et al. 1981); a 0.05 mag shift in zero-point and a significant color term are present. Koornneef (1983) incorporated the Engels et al. data plus 9 primary standards from Wamsteker (1981) as the only sources of M magnitudes in his Southern Hemisphere compilation. Campins et al. give $M$ magnitudes for only 4 of the primary Johnson standards, along with 11 main-sequence $\mathrm{G}$ stars.

When a comet is bright enough, the $5-\mu \mathrm{m}$ flux is usually observed with the bolometer along with the thermal emission at longer wavelengths. Thus, $M$ magnitudes of the bright bolometer standards need to be known relative to Vega and relative to the fainter InSb standards.

Apparently no work on $5-\mu \mathrm{m}$ bolometer standards for the Arizona system has been done since the early 1970s (Low and Rieke 1974), although the standard magnitudes at longer $\lambda$ have been revised and the present $M$ filter, used by Campins et al., differs from Johnson's (Table 2-1). The only other published bolometer standards are the Wyoming list (Gehrz et al. 1974). While Sinton and Tittemore (1985) measured a few of the bright standards on the InSb system, their magnitudes are systematically $\sim 0.1$ mag fainter than those of Low and Rieke or Gehrz et al. It is not clear whether the difference is due to differing effective wavelengths and/or different zero-points of the systems, or whether the InSb magnitudes were affected by non-linearity for these very bright stars. The late-type giants have strong $\mathrm{CO}$ bands within the $\mathrm{M}$ bandpass, so their behavior with different filter bandpasses cannot necessarily be predicted from simple blackbody curves.

The $10 \%$ difference between systems translates directly into a $10 \%$ difference in M-N color in the comet spectral energy distribution, affecting model calculations of the dust size distribution or the models of the surface thermal properties when a comet nucleus is 
directly detected. A renewed calibration of $5-\mu \mathrm{m}$ bolometer standards and a comparison of the instrument response functions on the Arizona, Wyoming, and MKO systems are clearly needed, if we are to obtain accurate measurements of the short-wavelength thermal flux from comets.

2.3.4 Standard Stars at 10 and $20 \mu \mathrm{m}$. Ideally, standard stars should be bright, nonvariable, and well distributed around the sky. In practice, these three criteria have not been fully met, for the brightest infrared stars tend to be variable M giants.

Comets have generally been observed on three photometric systems: Arizona, Mauna Kea (MKO), and Wyoming/Minnesota (WIRO). The A rizona system has been redefined by Rieke et al. (1985); the standard star magnitudes therein supersede Low and Rieke (1974). Tokunaga (1984) has reobserved a net of standard stars at the IRTF; these are the basis of the MKO system. The Wyoming standards are given in Gehrz et al. (1974) and Gehrz et al. (1987). As a further confusion, Vega is assigned a different magnitude in each of the three systems. All three systems are based on a broad $\mathrm{Q}$ filter at $20 \mu \mathrm{m}$, although the narrower $18-\mu \mathrm{m}$ filter is preferable to use, because of the better atmospheric transmission and the well-defined effective wavelength. We expect no differences between the 18 - and $20-\mu \mathrm{m}$ magnitudes for stars with no silicate dust.

The standard magnitudes on the three systems are compared in Table 2-5. This table

Table 2-5. Standard Star Magnitudes at $\mathrm{N}$ and $\mathrm{Q}$

\begin{tabular}{|c|c|c|c|c|c|c|c|c|}
\hline \multirow[b]{2}{*}{$\mathrm{BS}$} & \multirow[t]{2}{*}{ Star } & \multirow{2}{*}{$\begin{array}{c}\text { Spectral } \\
\text { Type }\end{array}$} & \multicolumn{3}{|c|}{$\mathrm{N}$} & \multicolumn{3}{|c|}{ Q } \\
\hline & & & ARIZ & IRTF & WIRO & ARIZ & IRTF & WIRO \\
\hline 7001 & $\alpha \mathrm{Lyr}$ & $\mathrm{AOV}$ & +0.02 & 0.00 & -0.03 & +0.02 & 0.0 & -0.03 \\
\hline 337 & $\beta$ And & MOIII & & -2.04 & -2.06 & & -2.09 & -2.11 \\
\hline 617 & $\alpha$ Ari & K2 III & -0.78 & & & -0.83 & & \\
\hline 1457 & $\alpha$ Tau & K5 III & -3.01 & -3.03 & -2.97 & -3.07 & -3.09 & -3.16 \\
\hline 1708 & $\alpha$ Aur & G8 III & -1.92 & -1.94 & & -1.96 & -1.93 & \\
\hline 2491 & $\alpha \mathrm{CMa}$ & A1 V & & -1.42 & & & -1.36 & \\
\hline 2943 & $\alpha \mathrm{CMi}$ & F5 IV & -0.72 & -0.76 & & -0.74 & -0.73 & \\
\hline 2990 & $\beta \mathrm{Gem}$ & KO III & -1.21 & -1.24 & -1.19 & -1.25 & -1.21 & -1.24 \\
\hline 3748 & $\alpha$ Hya & K3 III & -1.42 & & & -1.47 & & \\
\hline 4069 & $\mu \mathrm{UMa}$ & MO III & & -1.03 & -0.95 & & -1.08 & -1.01 \\
\hline 5340 & $\alpha$ Boo & K2 III & -3.15 & -3.17 & -3.15 & -3.19 & -3.13 & -3.20 \\
\hline 6705 & $\gamma$ Dra & K5 III & -1.48 & & & -1.54 & & \\
\hline 7525 & $\gamma \mathrm{Aql}$ & K3 II & -0.75 & -0.78 & & -0.80 & -0.82 & \\
\hline \multirow[t]{2}{*}{8775} & $\beta$ Peg(var) & M2 II & & -2.54 & -2.51 & & -2.61 & -2.80 \\
\hline & & & $-2.45^{a}$ & & & $-2.52^{a}$ & & \\
\hline
\end{tabular}

ARIZ: Arizona System, Rieke et al. (1985)

IRTF: Infrared Telescope Facility System, Mauna Kea, Tokunaga (1984)

WIRO: Wyoming Infrared Observatory and Univ. Minn., Gehrz et al. (1987)

a Tokunaga et al. (1986) 
should be referred to when Halley observations on the three systems are being compared. The Arizona and MKO systems agree to $0.01 \mathrm{mag}$ at $\mathrm{N}$ and $0.05 \mathrm{mag}$ at $\mathrm{Q}$ when the different zero points are accounted for. The WIRO magnitudes show a larger scatter; the differences could lead to a $10 \%$ difference in the $10 / 20 \mu \mathrm{m}$ comet color, depending on which standard was used.

Reobservation of a few stars that slow the largest scatter at $20 \mu \mathrm{m}$ and a comparison with the IRAS data at $25 \mu \mathrm{m}$ should lead to a set of $20-\mu \mathrm{m}$ standards self-consistent to $5 \%$ or better.

The main shortcoming of this list is the lack of reliable standards between RA $20 \mathrm{~h}$ and $1 \mathrm{~h}$ and at southern declinations. Particularly at $20 \mu \mathrm{m}$, where the atmosphere is the largest source of observational error, nearby standards are essential for accurate photometry.

With moderate effort, a common net of standard stars for use in the Northern Hemisphere could be developed.

2.3.5 Standards at 7.8 to $12.5 \mu \mathrm{m}$. Intermediate bandpass filters $(\lambda / \Delta \lambda \sim 10)$ covering the $10-\mu \mathrm{m}$ atmospheric window are useful for studying the silicate feature in comets when spectrophotometry is impractical because of time constraints and/or the faintness of the comet. Filters at $8.5,10.6$, and $12.5 \mu \mathrm{m}$ have been used for many years by Ney $(1974,1982)$ and colleagues at Minnesota to study comets, including Halley. A set of six filters from OCLI at $\lambda 7.8,8.7,9.8,10.3,11.6$, and $12.5 \mu \mathrm{m}$ have been employed for comet studies at the IRTF, UKIRT, and, recently, WIRO.

There has never been a careful calibration of standard stars through the OCLI filters. The only published standard magnitudes are those of the Wyoming system, measured through filters at $8.7,11.4$, and $12.5 \mu \mathrm{m}$ (Gehrz et al. 1974, 1987). Yet, standards accurate to $3 \%$ or better are quite feasible in the $10-\mu \mathrm{m}$ window, and would improve our ability to monitor temporal fluctuations in the strength of the silicate feature and search for weak features in short-period comets. The 7.8- and $12.5-\mu \mathrm{m}$ bandpasses are particularly important, in order to define the continuum level. Interpolation or extrapolation is not a viable means of obtaining accurate magnitudes; particularly given the $5 \%$ to $10 \%$ uncertainties at $\mathrm{M}$ and $\mathrm{Q}$ discussed above. From the WIRO list and from our IRTF photometry, it appears that the $\mathrm{K}$ giants brighten by $5 \%$ to $8 \%$ from $\lambda 8$ to $11.5 \mu \mathrm{m}$ and M0 giants by $\sim 10 \%$, relative to $\alpha$ Lyr or $\alpha \mathrm{CMa}$.

\subsection{POLARIZATION}

The polarization of light scattered by particles in the coma of a comet depends on scattering angle, composition of the particles, particle size distribution, shape, and surface roughness. These variables are of sufficient number and complexity that obtaining a unique solution is daunting. Observations spanning a wide range in wavelength can help to narrow the range of physical parameters. Thus, near-infrared polarimetry is a valuable addition to visual-wavelength data. Parameters such as the average particle albedo, average optical constants, and possible bulk composition have been estimated from infrared polarimetry of comet Halley by Brooke $e t$ al. (1987). Their results tend to be consistent with recent models of visible polarization data, although the models differ in specifics. 
A good discussion of the techniques of polarimetry is given by Serkowski (1974). The observations by Brooke et al. (1987) were made with a rotating wire grid polarizer inserted into the beam of the photometer. This technique, while simple, leads to a high instrumental polarization and high thermal background. A technique employed by newer polarimeters consists of a half-wave plate and a wire-grid polarizer inserted in the beam. In this design, the polarizer can be cooled and located inside the dewar. Rotating the half-wave plate provides the necessary polarimetric information. The instrumental polarization in such a system is close to zero.

\subsection{OBSERVING TECHNIQUE}

A discussion of photometric accuracy is not complete without mention of observing technique. The best photometric system is no guarantee of accurate photometry without careful attention to the way in which the data are taken. In particular, techniques for minimizing errors due to sky background fluctuations have to be employed.

Because the sky emission is usually brighter than the object under study, infrared photometry is generally carried out by rapidly differencing the signal centered on the object (object + sky) and the sky signal one or more beam diameters away. Telescopes designed for infrared work employ a wobbling secondary mirror for this purpose, at a frequency of 7 to $15 \mathrm{~Hz}$. For an extended source, such as the comet coma, the sky signal will still contain a contribution from the coma. 'Thus, the offset of the sky beam for comet observations has to be a compromise between the best signal-to-noise, which dictates a small offset, and the minimum contamination from the coma, which requires a large offset. A general rule under good sky conditions is to make the offset of the sky beam at least 2.5 beam diameters away for nucleus-centered observations; the coma contribution to the sky signal will then be less than $10 \%$ of the central brightness, assuming uniform radial outflow of dust.

Extinction should be measured every night, by observing standards over a range in air mass. Standards near the program objects should be used. Using mean extinction coefficients should be avoided whenever possible, since there is considerable scatter about the mean even at an excellent site such as Mauna Kea (Krisciunas et al. 1987). Humidity, seasonal variations in atmospheric aerosol content, and volcanic activity all play a role (Lockwood and Thompson 1986, Manduca and Bell 1979; Grasdalen et al. 1985).

\subsection{SUMMARY}

In summary, considerable progress towards an accurate, unified photometric system has been made since Morrison and Lebofsky (1979) noted 10\% to $15 \%$ discrepancies in their review of the calibration problems for asteroid radiometry. The absolute calibration of Vega is known to $\sim 3 \%$ at 1 to $5 \mu \mathrm{m}$ and better than $5 \%$ at $10 \mu \mathrm{m}$. The absolute calibration at $20 \mu \mathrm{m}$ is uncertain by $5 \%$ to $8 \%$. The Arizona photometric system has been brought up to date by Campins et al. (1985) and Rieke et al. (1985) for all of the standard 1- to $20-\mu \mathrm{m}$ bandpasses except $M(5 \mu \mathrm{m})$.

However, there are $10 \%$ systematic discrepancies between systems that need to be resolved, especially for the 5- $\mu \mathrm{m} M$ standards. An accurate measure of the thermal emission at $5 \mu \mathrm{m}$ is important for modeling the abundance of small hot grains. With moderate effort, 
a single uniform net of 5- to 20- $\mu \mathrm{m}$ standards can be achieved.

Color terms in the transformations between systems are the main problem for comet studies at J, H, and $\mathrm{K}$. A net of fainter $\mathrm{J}, \mathrm{H}, \mathrm{K}$ standards will be required for the next generation of infrared arrays. A common set of solar analog stars would be very helpful for comet color observations.

\section{Infrared Imaging}

Infrared imaging provides spatially resolved information that is unavailable at optical wavelengths, such as the characteristic dust temperature and particle size, the dust production rate, the distribution of silicate grains, the distribution of parent molecules, the presence of organic species, and the albedo of the dust grains.

\subsection{STATUS AT THE TIME OF HALLEY}

Infrared astronomy is now in the midst of a technological revolution that is centered on the development of 2-dimensional infrared arrays. In the past, such technical advances involved the development of new detectors, such as the germanium bolometer operated at $1.5 \mathrm{~K}$ (Low 1961) and the InSb photovoltaic detector (Hall et al. 1975), that led to orders-of-magnitude increases in sensitivity. Presently, we are witnessing a similar increase in the sensitivity per pixel in 2-dimensional infrared arrays that are as large as a $256 \times 256$ format (see summaries in Wynn-Williams and Becklin 1987; Tokunaga 1989).

The first infrared images of a comet were obtained in a scanning mode with the IRAS satellite at low spatial resolution (Walker et al. 1984). Ground-based maps soon followed, with $10-\mu \mathrm{m}$ maps of $\mathrm{P} /$ Giacobini-Zinner by Telesco et al. (1986) and Hayward and Grasdalen (1987). The former used a $4 \times 5$ bolometer array with $4-\operatorname{arcsec}$ resolution per pixel, while the latter used a single detector in a raster-scanning mode.

The latest 1 - to $5-\mu \mathrm{m}$ arrays were used to image Halley at $\mathrm{J}, \mathrm{H}, \mathrm{K}$, including a linear 128 element array (Hanner et al. 1987), a $32 \times 32 \mathrm{HgCdTe}$ array (Campins et al. 1989) and a $32 \times 32$ InSb array (Shure et al. 1989). Telesco et al. (1986), Hammel et al. (1987), and Hayward et al. (1988) have combined 10- $\mu$ m maps with optical images for Giacobini-Zinner and Halley to obtain albedo maps of the dust coma. This method can delineate regions of differing grain properties (size, composition).

\subsection{SOME TECHNOLOGICAL ASPECTS OF 2-DIMENSIONAL INFRARED ARRAYS}

In contrast to optical CCD arrays, which are monolithic devices, the best infrared arrays are hybrid devices. These devices are constructed in such a way that the multiplexer (the electronics that reads out the array) and the detector material (such as InSb or HgCdTe) are built separately and are then joined together. To accomplish this, indium bumps are attached to the multiplexer and the detector material, and the two pieces are pressed together. The indium bumps provide the electrical contact and mechanical support to keep the multiplexer and detector material together. This process, while seemingly delicate, leads to sturdy devices that can be repeatedly temperature cycled and also flight-qualified for space applications. Figure 2 shows how such an array is constructed. 


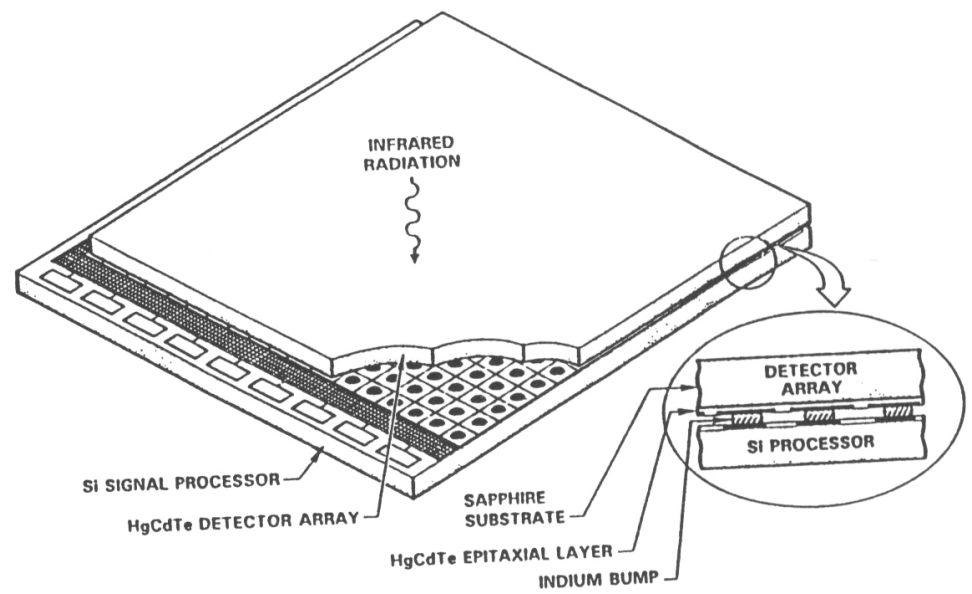

Fig. 2 Cross-section and perspective view of a hybrid infrared array. The detector material and multiplexer are manufactured separately, then attached with indium bumps, one per detector element. (from Rode et. al 1987).

The multiplexer is fabricated with standard silicon techniques, and thus it is possible to construct a simple readout amplifier for each pixel. Such devices are called "switched FET" or "direct readout" (DRO) devices. Although infrared detector material can be attached to a CCD multiplexer, the read noise is much higher than it is in a DRO device (Rode $e t$ al. 1987). In addition, the CCD multiplexer cannot be operated with good charge transfer efficiency at the very low temperatures required for infrared arrays (as low as $10 \mathrm{~K}$ ). Thus, all of the high-performance IR arrays now in use or under development utilize the DRO multiplexer.

The characteristics of some of the best 2-dimensional infrared arrays being used in astronomy are shown in Table 3-1. The low read noise and low dark current in these devices are leading to great advances in sensitivity, typically 3 to 5 magnitudes or more, depending on the application. While they are not yet generally available at observatories, in the next five years, it will be common to find such arrays in use. The application of these arrays for comet studies will have great impact.

To get an impression of how powerful these arrays can be if used on one of the 8-meterclass telescopes now being planned, we note that broad-band photometric observations at $2.2 \mu \mathrm{m}$ to a limit of $\mathrm{K} \approx 25 \mathrm{mag}$ will be possible. For spectroscopy with resolving power of 20,000 , it will be possible to reach a limit of $\mathrm{K} \approx 19 \mathrm{mag}$. The latter example is about 8 magnitudes fainter than is possible with current instrumentation on today's telescopes. Not only are read noise and dark current being lowered, but array dimensions are increasing. Within one year $256 \times 256 \mathrm{HgCdTe}$ arrays with read noise as low as 30 electrons may be possible (R. Thompson, private communication), and $256 \times 256 \mathrm{InSb}$ arrays with read noise as low as 40 electrons will be under development this year (D. Hall, private communication). 
Table 3-1. Selected IR Arrays With Highest Sensitivity.

\begin{tabular}{lccc}
\hline Spectral Range $(\mu \mathrm{m})$ & $1-2.5$ & $1-5$ & $5-14$ \\
Detector Material & HgCdTe & InSb & Si:Ga \\
Type & PV & PV & PC \\
Pixel Size & $60 \mu \mathrm{m}$ & $75 \mu \mathrm{m}$ & $75 \mu \mathrm{m}$ \\
Multiplexer Type & DRO & DRO & DRO \\
Format & $128 \times 128$ & $58 \times 62$ & $58 \mathrm{X} 62$ \\
Manufacturer & Rockwell & SBRC & SBRC \\
Quantum Efficiency & $70 \%$ & $45 \%$ & $30 \%$ \\
$\quad$ @ $\lambda(\mu \mathrm{m})=$ & 2.3 & 3.8 & 10.0 \\
Dark Current (elec./sec) & 9 & $<6$ & $<630$ \\
$\quad$ @ temperature $(\mathrm{K})=$ & 77 & 10 & 10 \\
Read Noise (elec.) & 50 & 280 & $<200$ \\
Full Well (elec.) & $3 \times 10^{5}$ & $10^{6}$ & $7 \times 10^{5}$ \\
& & & \\
Reference & 1 & 2 & 3 \\
\end{tabular}

a. $\mathrm{PV}=$ photovoltaic $; \mathrm{PC}=$ photoconductor .

b. $D R O=$ direct readout.

c. SBRC = Santa Barbara Research Corp.

d. References:

1 - M. Rieke, Univ. of Arizona, private communication.

2 - J. Pipher, Univ. of Rochester, private communication.

3 - D. Gezari et al. (1988). 
In addition, a $512 \times 512 \mathrm{PtSi}$ array is being tested for astronomical use in Tokyo (S. Sato, private communication). Such developments will lead to infrared astronomical instrumentation similar to that developed for visible wavelengths. Some astronomical instruments utilize linear reticon arrays, such as the infrared spectrometers described by Moorwood (1987) and Tokunaga et al. (1987). However, the relatively high read noise of these arrays ( $\sim 1000$ electrons) makes them unattractive for future instrumentation, and spectrometers incorporating 2-dimensional arrays such as those shown in Table 3-1 are already coming on line.

\subsection{FUTURE COMET SCIENCE}

The extraordinary sensitivity, combined with sub-arcsec spatial resolution, will allow exciting new comet science from ground-based observatories, such as:

- Imaging the spatial distribution of neutral molecular species to help identify and quantify any molecules originating from the surface of grains.

- Searching for the spectral signatures of icy grains and organic materials.

- Studying the volatile components during outbursts.

- Identifying volatiles in active comets far from the Sun.

- Tracing the spatial distribution for particles of different size or composition, based on their temperature, albedo, or spectral features.

- Deciding whether the steep spatial gradients observed in some comets can be explained by the sublimation of icy grains.

To take full advantage of the new technology for cometary studies, especially applications requiring absolute photometry, the same care must be taken in calibration, in defining instrument response function and effective wavelength, and in the choice of standards as we described in Section 2 for photometry by single detectors. A new net of fainter standard stars will be required for these sensitive instruments.

\section{Ground-Based Infrared Spectroscopy}

Infrared spectroscopy of comets has proven to be an extremely powerful tool for studying the composition of comets and physical processes in the coma of comets (see, for example, Crovisier 1989). The main reasons for this are: (1) the presence of many strong infrared rotational-vibrational bands from molecules, (2) the presence of diagnostic bands available only in the IR (such as the 3.4- $\mu \mathrm{m}$ carbonaceous band), and (3) the presence of strong dust emission or absorption bands (such as the 10- $\mu \mathrm{m}$ silicate band). A measure of the advances in recent years is the fact that the list of "observed species in comet spectra" by Wyckoff (1982) contains only one IR species: the 10- $\mu \mathrm{m}$ silicate band. In a mere six years, the number of species observed in the IR has been expanded to 10 (Campins and Tokunaga 1988; Crovisier 1989). The importance of IR spectroscopy in determining the composition 
of the cometary nucleus is also evident in the recent summary by Weaver (1989) on the volatile component in comets. IR spectroscopy is also providing the impetus for the study of physical processes such as the excitation of the water molecule (Bockelée-Morvan and Crovisier 1989; Weaver et al. 1986) and the ortho-para ratio of water (Mumma et al. 1988).

Present-day IR spectroscopy of comets is accomplished primarily with circular variable filter (CVF) spectrophotometers, grating spectrometers, and Fourier-Transform spectrometers (FTS). Until recently, IR spectroscopy has been carried out mostly by single-detector instruments or arrays of small detectors. For instance, infrared spectrometers utilizing multiplexed detector arrays have been operational only since about 1986 (Moorwood 1987; Tokunaga, Smith, and Irwin 1987; Lester et al. 1988); the IR array utilized consists of a linear array of 32 detectors. Prior to this time, infrared arrays consisted of discrete detectors mounted closely together (Witteborn and Bregman 1984; Wade 1983; Aitken and Roche 1982). One of the first IR spectrographs employing a 2-dimensional array was only recently announced as being available (Joyce 1989).

\subsection{CVF SPECTROPHOTOMETERS}

A CVF is an interference filter designed such that the wavelength transmitted varies as a function of position on the filter (Wolfe and Zissis 1978). The CVF is commonly formed as a $180^{\circ}$ sector; the film thicknesses vary with position around the circumference, and the disc is rotated to obtain the desired wavelength. A CVF is usually mounted in the filter wheel of a bolometer or InSb photometer, along with the standard broad-band filters. Thus it is possible to obtain a photometrically calibrated spectrum with a CVF. The resolving power, $\mathrm{R}=\lambda / \delta \lambda$, for this type of spectrometer is in the range of 30 to 100 .

Because of the simplicity of the method and the fact that only a single detector is required, the CVF spectrophotometer has been used since the 1960s (see, for example, Gillett et al. 1968 for a description of an early CVF spectrophotometer). The CVF operates like a photometer. To obtain a spectrum, the data are taken one wavelength at a time on both the object and the standard. If the airmass difference is large, then an extinction coefficient must be obtained for each wavelength. While simple, this process is time-consuming and is subject to various types of systematic errors, including guiding or tracking errors and nonuniform changes in the atmospheric transparency. Photometric weather is a requirement for this technique.

CVF spectrophotometers have been important tools in the detection of dust emission features. For example, the silicate emission band was clearly observed in the first 10$\mu \mathrm{m}$ CVF spectra of comets Bennett and Kohoutek (Hackwell 1971; Merrill 1974). Prior to Halley, the silicate emission feature in comets was thought to resemble spectra of amorphous silicates and the interstellar medium. However, the highest-resolution spectra of Halley, taken with a CVF (Campins and Ryan 1989) and a grating spectrometer (Bregman et al. 1987) show a well-defined peak at $11.3 \mu \mathrm{m}$, identified as crystalline olivine. As discussed by these authors, the silicate emission feature in comet Halley has a strong resemblance to that seen in interplanetary dust particles, strengthening the idea that at least some interplanetary dust particles originate from comets.

Another important result from CVF spectrophotometry was the discovery of organic material in comet Halley by the observation of an emission feature near $3.4 \mu \mathrm{m}$ by the 
infrared spectrometer on the VEGA 1 spacecraft (Combes et al. 1986; Moroz et al. 1987). The discovery was followed up with higher resolution spectrometers described below. The detailed discussion of these and other spectra is given by Encrenaz and Knacke (this volume).

\subsection{COOLED-GRATING SPECTROMETERS}

The increasing availability of IR arrays has made the use of cooled-grating spectrometers more common. Typically, the entrance aperture (or slit) and all of the optics after the entrance aperture are cooled to $77 \mathrm{~K}$ or lower, in order to eliminate the thermal emission from the instrument itself. Since the background is dispersed by the grating, each detector sees the background from only a single resolution element. Thus maximum sensitivity can be achieved in the $3-$ to $5-\mu \mathrm{m}$ spectral region, where ground-based IR instrumentation tends to be background-limited.

These instruments, which utilize 7 to 32 detectors in a linear format, are described by Aitken and Roche (1982), Wade (1983), Witteborn and Bregman (1984), Moorwood (1987), Tokunaga et al. (1987), Herter et al. (1987), and Glaccum et al. (1987). Grating spectrometers contributed important observations of Comet Halley at several ground-based telescopes and also on the Kuiper Airborne Observatory (KAO).

The principal advantages of multi-detector grating spectrometers over CVF spectrophotometers are: (1) the multiplex advantage of observing many resolution elements simultaneously, (2) the elimination or reduction of systematic errors arising from guiding and tracking errors and variable sky conditions, and (3) the availability of higher spectral resolution than achievable with CVFs (i.e., $R>100$ ). Advantage (2) is important: the reduction of systematic errors can be great and can make projects possible that would be impossible with a CVF spectrophotometer.

CVF and existing grating spectrometers are best suited for observations of broad emission features, such as those arising from dust grains. The major results obtained so far are the detection of carbonaceous material (near $3.4 \mu \mathrm{m}$ ), the detection of crystalline olivine in comet Halley, and the detection of broad emission features at 2.6 to $2.7,5.2,6.8,12.2,24.8$, and $28.4 \mu \mathrm{m}$ that are tentative or unidentified (Campins and Tokunaga 1988).

\subsection{FOURIER-TRANSFORM SPECTROMETERS (FTS)}

The operating principle of an FTS is described by Schnopper and Thompson (1974). Since the FTS is a multiplexing instrument, each detector receives all of the radiation transmitted by the instrument and filter. Thus, under conditions of high background, such as thermal wavelengths $(\lambda>3 \mu \mathrm{m})$, the FTS is at a sensitivity disadvantage compared with a cooledgrating spectrometer operating at the same wavelength. However, an FTS has the following very important advantages: (1) it can achieve very high spectral resolution, with $R=10^{5}$ or higher, (2) the instrument profile is known to high precision, and (3) the wavelength calibration is precisely known.

The most significant cometary work achieved with this technique has been the first detection of the $2.65-\mu \mathrm{m}$ transition of the water molecule in Comet Halley from the KAO (Mumma et al. 1986; Weaver et al. 1986), and a measurement of the ortho/para ratio of water (Mumma et al. 1988). In addition, Maillard et al. (1987) observed a hot band 
of water at $2.44 \mu \mathrm{m}$. An upper limit to the methane/water ratio of $4 \%$ for Halley was obtained by Drapatz et al. (1987) and a possible $3 \sigma$ detection of $4 \%$ for Wilson by Larson et al. (1989). Depending on the excitation temperature of the methane, these limits could be closer to $1 \%$ instead of $4 \%$ (Weaver 1989). These results are covered in detail by Weaver, Mumma, and Larson (this volume).

\subsection{SUMMARY}

As noted in the previous section, the advances in IR array technology are leading to an unparalleled increase in observing capability in IR astronomy. The advances in spectroscopy will be larger than those in imaging for ground-based observing. The reason for this is that the background emission from the telescope and sky will ultimately limit the sensitivity of ground-based imaging. Thus, as the read noise of the IR array becomes lower and lower, there will be a point where the background noise dominates and further reduction in read noise does not help. However, in the case of dispersive spectroscopy (such as with a cooledgrating spectrograph), the background emission can be greatly reduced. For high-resolution spectroscopy $(R=20,000)$ the instrument can be mostly detector-noise limited. Thus gains in sensitivity would continue with gains in detector performance. These points are discussed in detail by Ridgway and Hinkle (1988) and Tokunaga (1989). Gains in sensitivity of over 1000 in the next five years compared with existing spectroscopic instrumentation are now conceivable with current technology!

\section{Airborne and Space-Based IR Observations}

Although much can be learned about comets from ground-based observations, some investigations require a platform above the Earth's atmosphere.

\subsection{AIRBORNE OBSERVATIONS}

The Kuiper Airborne Observatory (KAO), flying at $12 \mathrm{~km}$, gives access to regions of the infrared spectrum containing signatures of known or suspected cometary constituents. Several of the new spectral features discovered in Halley were detected from the KAO, most notably the 2.65- $\mu \mathrm{m}$ water band (Mumma et al. 1986), through the use of FTS and grating spectrometers. Infrared photometry of comets was extended to $160 \mu \mathrm{m}$ with the Halley observations by Campins et al. (1987). A $6.8-\mu \mathrm{m}$ emission feature was seen in the Halley spectra of Bregman et al. (1987), possibly due to carbonates; no emission from organic molecules was evident in these 5 - to $8-\mu \mathrm{m}$ spectra.

Cooled grating spectrometers for the long-wavelength region, $\lambda>16 \mu \mathrm{m}$, are also used on the KAO. Spectrophotometry of Halley at 16 to $30 \mu \mathrm{m}$ (Herter et al. (1987) and 20 to $65 \mu \mathrm{m}$ (Glaccum et al. 1987) detected new features at $23.8 \mu \mathrm{m}$ and $28.4 \mu \mathrm{m}$, although the 16- to $22-\mu \mathrm{m}$ silicate feature was puzzling by its apparent absence. The $\mathrm{OH}$ rotational line at $119 \mu \mathrm{m}$ was observed with a Fabry-Perot spectrometer by Stacey et al. (1987).

The techniques applied in airborne photometry and spectroscopy do not differ in principle from ground-based techniques, in that sky chopping is employed and both photoconductors and bolometers are utilized. The main differences are in the production of far-infrared 
filters and the development of new types of detectors that are sensitive in the far-infrared.

SOFIA, a proposed 3-m airborne telescope, will allow detection of faint emission features from parent molecules and perhaps complex organic species. Fainter comets, and comets at larger heliocentric distances, will be observable. When combined with the sensitive new spectrometers described in Section 4, studies of the volatiles and ices in distant comets will be possible.

\subsection{OBSERVATIONS FROM EARTH ORBIT}

Airborne observations are hampered by atmospheric absorptions such as the $2.3-$ to $2.5-\mu \mathrm{m}$ $\mathrm{CO}$ bands, the $9.6-\mu \mathrm{m}$ ozone band, and the strong water bands beyond $30 \mu \mathrm{m}$. From orbiting platforms, the entire infrared spectrum will be accessible and the use of cryogenically cooled telescopes will lead to great gains in sensitivity. Continuous 7 - to $25-\mu \mathrm{m}$ spectra from Earth orbit will allow the continuum to be properly defined and the true shapes of both the 10- and $20-\mu \mathrm{m}$ silicate features to be recorded without telluric interference. Spectra of comets over a wide heliocentric distance range should tell us whether the presence of crystalline grains results from solar heating of the grains.

At 1 to $20 \mu \mathrm{m}$, emission from large grains is hidden by the emission from smaller, hotter grains. However, at long wavelengths, where the emissivity of the small grains drops, these grains can be detected and limits set on their abundance. With modest spatial resolution (10 to $20 \mathrm{arcsec}$ ), it may be possible to trace the spatial distribution of these large grains and the formation of dust trails. The first long-wavelength comet observations, obtained with the IRAS satellite, showed large-scale dust features, such as extended dust comae and dust trails.

ESA's Infrared Space Observatory (ISO), with a $60-\mathrm{cm}$ telescope, is scheduled to be launched in the early 1990s. The instrument complement includes grating spectrometers from $\lambda 3$ to $200 \mu \mathrm{m}$, IR cameras, a photopolarimeter, and a photometer. Instrumentation for NASA's Space Infrared Telescope Facility (SIRTF) is also under development. We look forward to exciting new comet discoveries from these and other space-based observations.

\section{Summary}

The state of infrared astronomy at the time of this review is rapidly changing from the use of single detector instruments to the use of 2-dimensional infrared arrays. Much has already been learned about the composition of comets and the nature of the dust in comets with these new instruments. Photometry will continue to play an important role in the study of comets, in order to measure the rate of dust production, define the continuum for high-resolution spectroscopic observations, give temporal coverage of highly variable comets such as Halley, and compare the dust properties among comets. Single-detector photometry centered on the coma can be carried out readily on 1-m-class telescopes, while spatially resolved photometry with array cameras will become standard on larger telescopes. Surprisingly, there is no unified infrared photometric system at this time, and this diminishes our ability to intercompare data sets. We have reviewed the infrared photometric systems so that comparisons can be made as best as is possible for the present. 
Infrared spectroscopic instruments have only recently used 2-dimensional infrared arrays, and these instruments have revealed new dust and molecular constituents in comets. Also, the use of the Kuiper Airborne Observatory has provided new information on comets at wavelengths unobservable from the ground. These developments, along with the development of infrared imaging with 2-dimensional arrays, offer the possibility of greatly enhancing our ability to study the composition of comets and physical conditions in the coma of comets.

We can only imagine with what interest the future scientific community will greet the arrival of Comet Halley in the year 2061 or what the state of infrared techniques will be then. While we are less than satisfied with our incomplete knowledge and glimpses of the nature of comets, perhaps our successors in that distant epoch will appreciate our struggles and find our data useful (especially that in the International Halley Watch archive) even while imperfect.

The authors would like to thank Roger Knacke and Humberto Campins for helpful discussions. M. Hanner's work was carried out at the Jet Propulsion Laboratory, California Institute of Technology, under contract with the National Aeronautics and Space Administration. A. Tokunaga acknowledges the support of NASA Contract NASW-3159.

\section{REFERENCES}

A'Hearn, M.F. (1988). 'Observations of cometary nuclei,' Ann. Rev. Earth Planetary Sci. 16, 273-293.

A'Hearn, M.F., Dwek, E., and Tokunaga, A.T. (1984). 'Infrared photometry of Comet Bowell and other comets.' Astrophys. J. 282, 803.

Aitken, D.K., and Roche, P.F. (1982). ' $8-13 \mu$ m spectrophotometry of compact planetary nebulae and emission line objects.' Mon. Not. R. Astr. Soc. 200, 217.

Allen, D.A., and Cragg, T.A. (1983). 'The AAO JHKL' photometric standards.' MNRAS 203, 777-783.

Becklin, E.E., and Westphal, J.A. (1966). 'Infrared Observations of Comet 1965f.' Astrophys. J. $145,445$.

Beichman, C., et al. (1985). 'IRAS Explanatory Supplement,' Chapter VI.

Blackwell, D.E., Leggett, S.K., Petford, A.D., Mountain, C.M., and Selby, M.J. (1983). 'Absolute calibration of the infrared flux from Vega at $1.24,2.20,3.76$, and $4.6 \mu \mathrm{m}$ by comparison with a standard furnace.' MNRAS 205, 897-905.

Bockelée-Morvan, D., and Crovisier, J. (1989). 'The nature of the $2.8-\mu \mathrm{m}$ enission feature in cometary spectra.' Astron. Astrophys. 216, 278.

Booth, A.J., Selby, M. J., Blackwell, D. E., Petford, A. D., and Arribas, S. (1989). 'Determination of the absolute flux from Vega at $2.25 \mu \mathrm{m}$.' Astron. Astrophys. 218, 167.

Bregman, J.D., Campins, H., Witteborn, F.C., Wooden, D.H., Rank, D.M., Allamandola, L.J., Cohen, M., and Tielens, A.G.G.M. (1987). 'Airborne and groundbased spectrophotometry of comet P/Halley from 5-13 micrometers.' Astron. Astrophys. 187, 616.

Brooke, T.Y., Knacke, R.F., and Joyce, R.R. (1987). 'The near-infrared polarization and color of comet P/Halley, Astron. Astrophys. 187, 621.

Campins, H., Joy, M., Harvey, P.M., Lester, D.F., and Ellis, H.B. (1987). 'Airborne photometry of Comet Halley from 40 to 160 microns.' Astron. Astrophys. 187, 632.

Campins, H., Rieke, G.H., and Lebofsky, M.J. (1985). 'Absolute calibration of photometry at 1 through $5 \mu \mathrm{m}$.' Astron. J. 90, 896. 
Campins, H., Bregman, J.D., Witteborn, F.C., Wooden, D.H., Rank, D.M., Allamandola, L.J., Cohen, M., and Tielens, A.G.G.M. (1987). 'Airborne spectrophotometry of comet Halley from 5 to 9 microns.' In Proc. 20th ESLAB Symp. on the Exploration of Halley's Comet (B. Battrick, E.J. Rolfe, and R. Reinhard, Eds.), ESA SP-250, Vol. 2, p. 121.

Campins, H., and Tokunaga, A., (1988). 'Infrared observations of the dust coma.' In Infrared Observations of Comets Halley and Wilson and Properties of the Grains, NASA Conf. Pub. 3004 (M.S. Hanner, Ed.), p. 1.

Campins, H., Rieke, M. J., and Rieke, G. H. (1989). 'An infrared color gradient in the inner coma of Comet Halley.' Icarus 78, 54.

Campins, H., and Ryan, E.V. (1989). 'The identification of crystalline olivine in cometary silicates.' Astrophys. J. 341, 1059.

Carter, B.S. (1990). 'Southern JHKL standards.' MNRAS 242, 1-5.

Combes, M., et al. (1986). 'Infrared sounding of comet Halley from VEGA 1.' Nature 321, 266.

Crovisier, J. (1989). 'Infrared cometary spectroscopy.' In 22nd ESLAB Symp. on Infrared Spectroscopy in Astronomy, ESA SP-290, in press.

Drapatz, S., Larson, H.P., and Davis, D.S. (1987). 'Search for methane in comet P/Halley.' Astron. Astrophys. 187, 497.

Dreiling, L.A., and Bell, R.A. (1980). 'The chemical composition, gravity and temperature of Vega.' Astrophys. J. 241, 736.

Elias, J.H., Frogel, J.A., Matthews, K., and Neugebauer, G. (1982). 'Infrared standard stars.' Astron. J. 87, 1029.

Elias, J.H., Frogel, J.A., Hyland, A.R., and Jones, T.J. (1983). 'Comparison of the Mt. Stromlo/ AAO. and Cal Tech/Tololo infrared photometric systems.' Astron. J. 88, 1027.

Engels, D., Sherwood, W.A., Wamsteker, W., and Schultz, G.V. (1981). 'Infrared observations of southern bright stars.' Astron. Astrophys. Suppl. Ser. 45, 5.

Gehrz, R.D. Grasdalen, G.L., and Hackwell, J.A. (1987). 'Infrared astronomy.' In Encyclopedia of Physical Science and Technology 2, 53.

Gehrz, R.D., Hackwell, J.A., and Jones, T.W. (1974). 'Infrared observations of Be stars from 2.3 to 19.5 microns.' Astrophys. J. 191, 675 .

Gezari, D.Y., Folz, W.C., Woods, L.A., and Woolridge, J.B. (1988). 'A 58 x 62 pixel Si:Ga array camera for 5-14 $\mu \mathrm{m}$ astronomical imaging.' Proc. SPIE. 973, in press.

Gillett, F.C., Dereniak, E.L., and Joyce, R.R. (1977). 'Detectors for infrared astronomy.' Opt. Engr. 16, 544.

Gillett, F.C., Low, F.J., and Stein, W.A. (1968). 'Stellar spectra from 2.8-14 microns.' Astrophys. J. 154, 677 .

Glaccum, W., Moseley, S.H., Campins, H., and Loewenstein, R.F. (1987). 'Airborne spectrophotometry of $\mathrm{P} /$ Halley from 20 to 65 microns.' Astron Astrophys. 187, 635.

Glass, I.S. (1974). 'JHKL photometry of 145 southern stars.' Mon. Not. Astr. Soc. S. Africa 33, 53.

Grasdalen, G. L., Gehrz, R.D., Hackwell, J.A., and Freedman, R. (1985). '20-micron transparency and atmospheric water vapor at the Wyoming infrared observatory.' Pub. Astron. Soc. Pac. 97, 1013.

Hackwell, J.A. (1971). 'Emission spectrum of comet Bennett.' Observatory 91, 33.

Hall, D.N.B., Aikens, R.S., Joyce, R., and McCurnin, T.W., (1975). 'Johnson noise limited operation of photovoltaic InSb detectors.' App. Optics 14, 450.

Ilammel, H.B., Telesco, C.M., Campins, H., Decher, R., Storrs, A.D., and Cruikshank, D. P. (1987). 'Albedo maps of comets P/Halley and P/Giacobini-Zinner.' Astron. Astrophys. 187, 665.

Hanner, M.S. (1984). 'A comparison of the dust properties in recent periodic comets.' Adv. Space Res., 4, (9), 189. 
Hanner, M.S., Tokunaga, A.T., Veeder, G.J., and A'Hearn, M.F. (1984). 'Infrared photometry of the dust in comets.' Astron. J. 89, 162.

Hanner, M.S., Kupferman, P.N., Bailey, G., and Zarnecki, J.C. (1987). 'Infrared imaging with JPL's linear array camera.' In Infrared Astronomy with Arrays (C.G. Wynn-Williams and E.E. Becklin, Eds.), Institute for Astronomy, Univ. of Hawaii, Honolulu, p. 205.

Hanner, M.S., Ed. (1988). 'Infrared observations of Comets Halley and Wilson and properties of the grains,' NASA Conference Publ. 3004.

Hayward, T.L., and Grasdalen, G.L. (1987). 'Infrared images of comets. I. P./Giacobini-Zinner (1985e).' Astron. J. 94, 1339.

Hayward, T.L., Grasdalen, G.L., and Green, S.F., (1988). 'An albedo map of P/Halley on 13 March 1986.' In Infrared Observations of Comets Halley and Wilson and Properties of the Grains, NASA Conf. Pub. 3004 (M.S. Hanner, Ed.), p. 151.

Hayes, D.S. (1985). 'Stellar absolute fluxes and energy distributions from 0.32 to $4.0 \mu \mathrm{m}$.' In IAU Symp. 111, Calibration of Fundamental Stellar Quantities (D.S. Hayes, L.E. Pasinetti, and A.G. D. Philip, Eds.), Reidel, Dordrecht, p. 225.

Herter, T., Campins, H., and Gull, G.E. (1987). 'Airborne spectrophotometry of P/Halley from 16 to 30 microns.' Astron. Astrophys. 187, 629.

Johnson, II.L. (1966). 'Astronomical measurements in the infrared,' Ann. Rev. Astron. Ap. 193.

Johnson, II.L. (1965). 'The absolute calibration of the Arizona photometry,' Comm. Lunar Plan. Lab 3, 73.

Johnson, H.L. (1965). 'Interstellar extinction in the galaxy,' Astrophys. J. 141, 923.

Johnson, H.L., et al. (1966). Comm. Lunar Plan. Lab 4, 99.

Jones, T.J., and Hyland, A.R. (1982). 'Multiaperture JHK photometry of the globular clusters in the Fornax dwarf spheroidal galaxies.' Mon. Not. Roy. Astr. Soc. 200, 509.

Joyce, R. (1989). 'Availability of KPNO IR spectrometer (CRSP).' NOAO Newsletter No. 17, p. 22.

King, I. (1952). 'A note on the concept of effective wavelength.' Astrophys. J. 115, 580.

Koornneef, J. (1983). 'Near infrared photometry I.' Astron. Astrophys. Suppl. Ser. 51, 489.

Koornneef, J. (1983). 'Near infrared photometry II.' Astron. Astrophys. 128, 84-93.

Krisciunas, K., Sinton, W., Tholen, D., Tokunaga, A., Golisch, W., Griep, D., Kaminski, C., Impey, C., and Christian, C. (1987). 'Atmospheric extinction and night sky brightness at Mauna Kea.' Pub. Astron. Soc. Pac. 99, 887.

Kurucz, R.L. (1979). 'Model atmospheres for G, F, A, B and O stars'. Ap.J. Suppl, 40, 1.

Larson, H.P., Weaver, H.A., Mumma, M.J., and Drapatz, S. (1989). 'Airborne infrared spectroscopy of comet Wilson (19861) and comparisons with comet Halley.' Astrophys. J., submitted.

Lester, D.F., Harvey, P.M., and Carr, J. (1988). 'Properties of the gas and stellar content of the superluminous galaxy NGC 6240.' Astrophys. J. 329, 641.

Lockwood, G.W., and Thompson, D.T. (1986). 'Atmospheric extinction - the ordinary and volcanically induced variations, 1972-1985.' Astron. J. 92, 976.

Low, F.J. (1961). 'Low-temperature germanium bolometer.' J. Opt. Soc. Am. 51, 1300.

Low, F.J., and Rieke, G.H. (1974). 'The instrumentation and techniques of infrared photometry.' In Methods of Experimental Physics, Vol. 12 (N. Carelton, Ed.), Academic Press, N.Y., pp. 415-452.

Manduca, A., and Bell, R.A. (1979). 'Atmospheric extinction in the near-infrared.' Pub. Astron. Soc. Pac. 91, 848.

Merrill, K.M. (1974). '8-13 $\mu \mathrm{m}$ spectrophotometry of comet Kohoutek.' Icarus 23, 566.

Moorwood, A.F.M. (1987). 'IRSPEC: Design, performance and first scientific results.' In Infrared Astronomy with Arrays (C.G. Wynn-Williams and E.E. Becklin, Eds.), Univ. of Hawaii, Honolulu, p. 379. 
Moroz, V.I., et al. (1987). 'Detection of parent molecules in comet P/Halley from the IKS-Vega experiment.' Astron. Astrophys. 187, 513.

Morrison, D., and Lebofsky, L. (1979). 'Radiometry of asteriods.' In Asteroids (T. Gehrels, Ed.), Univ. Arizona Press, Tucson, p. 184.

Mountain, C.M., Leggett, S.K., Selby, M.J., Blackwell, D.E., and Petford, A.D. (1985). 'Measurement of the absolute flux from Vega at $4.92 \mu \mathrm{m}$.' Astron. Astrophys. 151, 399-402.

Mumma, M.J., Weaver, H.A., Larson, H.P., Davis, D.S., and Williams, M. (1986). 'Detection of water vapor in Halley's comet.' Science 232, 1523.

Mumma, M.J., Blass, W.E., Weaver, H.A., and Larson, H.P. (1988). 'Measurements of the orthopara ratio and the nuclear spin temperature of water vapor in comets Halley and Wilson (19861) and implications for their origin and evolution.' BAAS 20, 826; Proc. Workshop on Formation and Evolution of Planetary Systems, STScI, May 9-11, 1988.

Neckel, H., and Labs, D. (1981). 'Improved data of solar spectral irradiance from 0.33 to $1.25 \mu \mathrm{m}$.' Solar Phys. 74, 231.

Ney, E.P. (1974). 'Multiband photometry of comets Kohoutek, Bennett, Bradfield, and Encke.' Icarus, $23,551$.

Ney, E.P. (1982). 'Optical and infrared observations of comets in the range $0.5 \mu \mathrm{m}$ to $20 \mu \mathrm{m}$.' In Comets (L.L. Wilkening, Ed.), Univ. Arizona Press, Tucson, p. 323.

Rieke, G.H., Lebofsky, M.J., and Low, F.J. (1985). 'An absolute photometric system at 10 and 20 $\mu \mathrm{m}$.' Astron. J. 90, 900.

Ridgway, S.T., and Hinkle, K.H. (1988). "The impact of array detectors on high resolution infrared spectroscopy.' In The Impact of Very IIigh S/N Spectroscopy on Stellar Physics (G. Cayrel de Strobel and M. Spite, Eds.), p. 61.

Rode, J.P., Blackwell, J.D., Blessinger, M.A., and Vural, K. (1987). 'SWIR HgCdTe focal plane arrays for astronomy.' In Infrared Astronomy with Arrays (C.G. Wynn-Williams and E.E. Becklin, Eds.), Institute for Astronomy, Univ. of Hawaii, Honolulu, p. 13.

Schnopper, H.W., and Thompson, R.I. (1974). 'Fourier spectrometers.' In Methods of Experimental Physics, Vol. 12, Part A (N. Carleton, Ed.), Academic Press, N.Y., p. 491.

Serkowski, K. (1974). 'Polarization techniques.' In Methods of Experimental Physics, 12 (N. Carleton, Ed.) Academic Press, N.Y., p. 361.

Simpson, J.P., Cuzzi, J.N., Erickson, E.F., Strecker, D.W., and Tokunaga, A.T. (1981). 'Mars: Far-infrared spectra and thermal emission models.' Icarus 48, 230-245.

Shure, M., Nagata, T.M., Tokunaga, A.T., Forrest, W.J., Pipher, J.L., and Woodward, C.E. (1989). 'Ground-based infrared imaging of comet Halley.' In preparation.

Sinton, W.M., and Tittemore, W.C. (1984). 'Photometric standard stars for L' and M filter bands.' Astron. J. 89, 1366.

Stacey, G.J., Lugten, J.B., and Genzel, R. (1987). 'Detection of $\mathrm{OH}$ rotational emission from comet $\mathrm{P} /$ Halley in the far-infrared.' Astron. Astrophys. 187, 451.

Telesco, C.M., Decher, R., Baugher, C., Campins, H., Mozurkewich, D., Thronson, H.A., Cruikshank, D.P., Hammel, H.B., Larson, S., and Sekanina, Z. (1986). 'Thermal-infrared and visual imaging of comet Giacobini-Zinner.' Astrophys. J. Lett. 310, L61.

Tokunaga, A.T. (1989). 'Infrared detector arrays and some applications to spectroscopy.' Ap. Space Sci. 160, 333.

Tokunaga, A.T. (1986). The NASA Infrared Telescope Facility Photometry Manual.

Tokunaga, A.T. (1984). 'A reevaluation of the 20 magnitude system.' Astron. J. 89, 172.

Tokunaga, A.T., Golisch, W.F., Griep, D.M., Kaminski, C.D., and Hanner, M.S. (1986). 'The NASA infrared telescope facility Comet Halley monitoring program. I. Preperihelion results.' Astron. J. 92, 1183.

Tokunaga, A.T., Smith, R.G., and Irwin, E. (1987). 'Use of a 32-element Reticon array for 1 to 5 micrometer spectroscopy.' In Infrared Astronomy with Arrays (C.G. Wynn-Willians and E.E. Becklin, Eds.), Univ. of Hawaii, Honolulu, p. 367. 
Wade, R. (1983). 'A 1-5 micron cooled grating array spectrometer and Fabry-Perot system for the UKIRT.' Proc. SPIE 445, 47.

Walker, R.G., Aumann, H.H., Davies, J., Green, S., De Jong, T., Houck, J.R., and Soifer, B.T. (1984). 'Observations of comet IRAS-Araki-Alcock 1983d.' Astrophys. J. 278, L11.

Wamsteker, W. (1981). 'Standard stars and calibration for JHKLM photometry,' Astron. Astrophys. 97, 329 .

Weaver, H.A. (1989). 'The volatile composition of comets.' In Highlights of Astronomy, 8, 387.

Weaver, H.A., Mumma, M.J., Larson, H.P., and Davis, D.S. (1986). 'Post-perihelion observations of water in comet Halley.' Nature 324, 441.

Witteborn, F.C., and Bregman, J.D. (1984). 'A cryogenically cooled, multidetector spectrometer for infrared astronomy.' Proc. SPIE 509, 123.

Wolfe, W.L., and Zissis, G.J. (1978). The Infrared Handbook, p. 7-118.

Wright, E.L. (1976). 'Recalibration of the far-infrared brightness temperatures of the planets.' Astrophys. J. 210, 250.

Wyckoff, S. (1982). 'Overview of comet observations.' In Comets (L.L. Wilkening, Ed.), Univ. of Arizona Press, Tucson, p. 3.

Wynn-Williams, C.G., and Becklin, E.E., Eds. (1987). Infrared Astronomy with Arrays, Proc. Workshop on Ground-Based Astronomical Observations with Infrared Array Detectors. Institute for Astronomy, Univ. of Hawaii, Honolulu. 